\title{
De la genèse du corps poétique dans la genèse de «Lecture du corps »
}

Ayant déjà montré par le passé combien la naissance à la poésie passait indubitablement par une dimension corporelle dans l'œuvre de Bauchau ${ }^{1}$, nous souhaitions poursuivre cette analyse à l'aune de l'étude de ses manuscrits. En effet, comme le rappelle Geneviève Henrot ${ }^{2}$, la poésie est une expérience corporelle au cours de laquelle le corps est moins un instrument d'écriture qu'objet même de la composition poétique. Si le corps apparait dans sa poésie comme origine essentielle de l'écriture, qu'en est-il dans le processus créateur? Dans quelle mesure le corps, élément matériel participe-t-il à la création poétique bauchalienne, fondée sur l'exploration des profondeurs inconscientes de l'humain? Comment se concilient dans l'élaboration poétique le corps et l'impalpable inconscient ? Le poème est-il subi par le poète et subit pour le poète quand celui-ci l'inscrit dans la réalité de la conscience par la corporéité qu'il lui donne? Nous avons choisi de nous consacrer à un recueil fondamental pour cette perspective : La Chine intérieure. Dans la mesure où «Lecture du corps» annonce la perception fragmentaire et collective du corps de «La Chine intérieure», notre attention s'est focalisée finalement sur cette unique partie du recueil. Il est celui qui offrait également un plus grand nombre de manuscrits à explorer. Le corps en blason de la version finale et publiée n'est pas entièrement créé dans la mesure où son assemblage est en discontinu. Cette corporéité poétique se traduit-elle par un phénomène de corporéisation lors du processus créateur? Prendre possession d'un corps est concomitant à l'assimilation de ce dernier à un paysage. Par ailleurs, la symbiose avec les éléments naturels et l'inscription élémentaire du corps dans l'espace est un des leitmotivs de la poésie bauchalienne. Notre étude se concentrera essentiellement sur certains poèmes de cette section dans la mesure où

\footnotetext{
${ }^{1}$ Marianne Froye, «Du corps du poète au corps poétique dans Heureux les déliants », Catherine Mayaux et Myriam Watthee-Delmotte (dir.), Henry Bauchau. Écrire pour habiter le monde, Presses Universitaires de Vincennes, 2009, pp. 111-123.

${ }^{2}$ Geneviève Henrot, Henry Bauchau poète. Le Vertige du seuil, Genève, Droz, 2003.
} 
pour: «L'écoutée », «Le lierre de Parc Trihorn», «L'ardoise», «Les yeux d'Argile », "Val de Mérence », «La corde à mon arc » et «L'enfant de sel », il n’y a pas d'état intermédiaire. Les états successifs des poèmes montreront comment se constitue le corps du poème, mais aussi comment il se fait matière et gagne en matérialité. Mais cette existence reste malgré tout problématique, car elle se dit dans le silence de la page blanche, la signifiance n'est audible du lecteur que dans l'absence de la voix du poète. Pour mener à bien notre étude, nous nous appuierons sur l'étude des manuscrits et des tapuscrits conservés au Fonds Henry Bauchau de l'Université catholique de Louvain numérotés de A375 à A484 et sur les éditions successives de «Lecture du corps » chez Seghers en 1974 (CI), chez Actes Sud en 1986 (P), chez Labor en 1995 (HD) et Actes Sud en 2009 (PC).

\section{À l'origine du corps du poème}

L'importance de la psychanalyse dans la vie de Bauchau n'est plus à rappeler, cependant dans l'optique d'étudier les manuscrits de cet auteur, il est légitime de s'interroger sur l'origine du poème et sa manifestation. N'est-il que le produit d'une écriture automatique qui jaillit sur la page blanche et à laquelle le poète ne soumet jamais sa conscience? Dans ce cas, l'étude des manuscrits risque d'être rapidement stérile car peu significative du fait d'une version unique du poème. Or, le volume des manuscrits et des tapuscrits manifeste le travail de l'artisan-poète. Comment se concilient dans la création poétique bauchalienne deux forces antinomiques : celle de l'inconscient et celle du labeur.

\section{De l'ouverture à la clôture du poème}

Les différents états de plusieurs poèmes soulignent cette lutte dans la création poétique de la raison ou de la conscience et de l'ouverture à des profondeurs inconnues. L'effacement du Je comme sujet conscient dans différentes versions de plusieurs poèmes devient le signe de la lutte entre la raison et l'ouverture à l'inconscient lors de la composition d'une œuvre. Paradoxalement, la disponibilité aux profondeurs de l'enfance et à celles de son moi nécessite l'intervention raisonnée du poète. C'est en travaillant le poème que progressivement Bauchau s'offre pleinement à son intériorité. L'effacement du Je pourrait alors apparaitre conscient de la part du poète, l'état de ses différents manuscrits manifestant un travail - travail sur soi comme travail sur le poème en train de s'élaborer. La 
démarche paradoxale de fouiller son inconscient de manière consciente pour que le poème s'élève de l'obscurité enfouie en soi devient un trait caractéristique de son écriture. Pour "Les mains limpides", «L'entreprise du sommeil » et «Les paumes », les états successifs de ces trois poèmes montrent une lutte entre la raison et l'inconscient du poète. Le premier combat à mener est la disparition du père dans les poèmes. Symbole de l'ordre et de la voix de la raison, le père, présent initialement dans le texte disparaît progressivement dans les versions ultérieures, comme si la voix de la raison s'effaçait au fur et à mesure que le poème montait à la conscience du poète. Ainsi, dans les premières versions des « Mains limpides » (A394-398), le couple parental est présent avec
Mère des mains lucides
terre formée
de la matière même
du soleil
Limpide
Est le courage du bonheur
Délivre-nous
De la raison du père

Si dans la seconde version manuscrite (A396-397), «Limpide / est le courage du bonheur» disparait, «la raison / du père» est toujours présente en deux vers au lieu d'un seul comme pour signifier que la mère avait la tâche par sa fonction poétique de délivrer à la fois du père et de la raison, les deux étant en tous les cas nocifs à la parole poétique. Le premier tapuscrit (A398) reprend le vers éliminé et fait du verbe à l'impératif et de son complément un seul vers. Les versions ultérieures hésitent entre garder et supprimer ces deux vers. La version finale les a définitivement supprimés. Il n'est pas innocent de la part de Bauchau d'associer le père à la raison, nous devons entendre toute la portée psychanalytique de ce rapprochement. Il n'est pas non plus anodin de sa part d'en appeler à la mère, malgré la lutte qu'il doit mener. Or, la fabrication du poème passe par l'élimination de la conscience pour s'offrir librement et consciemment à la terre mère. L'hésitation entre «obéir » et «consentir » quelques vers auparavant dans ce même poème participe de la même mouvance. Dans la version manuscrite (A394), quatre vers se succèdent: "Je n'ai pas obéi / à ma fertilité / Je n’ai pas consenti / à la sainteté des matières » et deviendront dans la version 
finale «Je n’ai pas consenti à ma fertilité ». Le choix du verbe consentir, le transfert des compléments accentuent le chemin vers l'ouverture aux forces créatrices du poème. La raison obligée abdique pour laisser place à une force supérieure indépendante de son moi. Les premiers tapuscrits de «L'entreprise du sommeil» font état de la même lutte entre «Mérence» et la «pauvreté du père» que la version finale aura définitivement abandonnée après l'avoir laissée un temps, notamment dans l'édition de 1974 chez Seghers. Lutte à rapprocher de la fin du vers médian aux accents ô combien rimbaldiens « on était pensé ». Le poète doit se battre consciemment contre lui-même pour laisser émerger la part inconsciente propice à la création poétique. C'est ce que manifestent sous une autre forme «Les paumes » nommées initialement «Les doigts de la main » avec la triade corps/esprit/cœur abandonnée au profit de la dyade corps/esprit.

Dire l'immatérialité poétique par la matérialité du monde et du corps passe nécessairement pour Bauchau par la dissolution du Je. Le poète élimine tous les freins à la création : la raison et le père, principalement, mais pour favoriser la venue du poème, il faut que lui-même s'extraie du poème, qu'il ne soit plus un objet de conscience dans le texte. Le texte doit venir à lui, il doit subir le poème et non tenter d'en forcer l'arrivée. Ce sont ainsi plusieurs poèmes qui manifestent ce retrait comme «Le second souffle » (A438) saturé du pronom je sujet avec « je sais » à trois reprises en début de poème et « je te respire », « je te salue » par la suite. Seul restera le «Je sais / mais qui sait ça » final. De même, le manuscrit des «Inscriptions » fait état d'une répétition de « je me souviens » et de «j'ai mémoire». Seul restera «j’ai mémoire». Le changement grammatical du sujet poétique souligne le dessaisissement de lui-même qui habite le poète pour que le poème advienne. Le Je doit disparaitre pour que la matière poème s'élabore.

\section{Du minéral au végétal}

Ce processus prend un sens très concret quand il en appelle au minéral et au végétal pour exister. Comme l'a montré Corina Dambean dans sa thèse ${ }^{3}$ et dans un article consacré au séjour suisse $e^{4}$ de Bauchau, le

\footnotetext{
${ }^{3}$ Corina Dambean, L'Imaginaire du minéral dans l'auvre d'Henry Bauchau, sous la direction de Catherine Mayaux et Myriam Watthee-Delmotte, Université de Cergy-Pontoise, 2012.
} 
minéral participe à la relation entre le sujet et le monde, il lui permet de s'intégrer en faisant sens entre les parties. Comme nous l'avons nousmême montré dans cette même revue ${ }^{5}$, «la naissance à la poésie [...] est symptomatique d'un traitement particulier des éléments : ils sont la voie d'accès poétique au monde pour cet auteur $»^{6}$. Or, les manuscrits confirment le cheminement du poète vers les éléments. L'évolution de la place de l'argile est primordiale pour comprendre l'élaboration élémentaire de la poésie bauchalienne. Les caractéristiques terreuses, imperméables et plastiques de l'argile deviennent le symbole de l'effort du poète et de son travail sur sa propre matière en résonance avec le monde lors de la venue d'un poème. Argile, symbole parfois déifié de la femme instigatrice de la poésie très présente dans les premiers états des manuscrits, disparaît comme mot au profit de ses propriétés qui infusent le poème final. Elle permet de quitter le minéral pour s'acheminer doucement vers le végétal, de la dureté de la matière résistante vers la fécondité poétique. L'état initial du «Convive d'octobre » insiste dans ses derniers vers sur la propriété déliante de l'argile pour finalement ne conserver qu'un terme beaucoup plus générique avec «Toute ma terre déliée écoute son érable rouge » (HD, p. 150). De même dans «Les pieds sur terre », le jeu de mots facile sur le colosse aux pieds d'argile, sur la « terre agile » et la «Sibylle/argile » est abandonné pour ne garder dans la version finale que «terre agile/Sibylle». La proximité sonore est suffisamment grande pour faire entendre dans une syllepse phonique les deux termes. Le colosse est remplacé par une écholalie «nos pas/nos paradis » plus efficace pour joindre la réussite poétique à la matière monde. C'est un effacement progressif qui se poursuit jusque dans les publications ultérieures, puisque l'édition de 2009 modifie le titre et le leitmotiv «Les yeux d'argile» en «Les yeux de Laure». La correspondance dite parfois, mais supposée souvent entre la femme aimée et l'argile est achevée et confirmée avec cette modification. Le jeu sur les majuscules l'anticipait également. L'évolution du titre du dernier poème «La signature » intitulé initialement «Les sons d'argile » (A482)

\footnotetext{
${ }^{4}$ Corina Dambean, «Paysage suisse et imaginaire minéral chez Henry Bauchau », dans Revue internationale Henry Bauchau, n³, 2010, pp. 91-100.

${ }^{5}$ Marianne Froye, "Présence lexicale de la Suisse dans la poésie d'Henry Bauchau de 1951 à $1975 »$, Ibid., pp. 77-90.

${ }^{6}$ Ibid., p. 80.
} 
puis «L'existence d'argile» rappelle une des origines fondamentales du poème : la femme aimée.

\title{
La matière-poème
}

Le poème prend forme dans et par la matière, une interaction entre les deux prend même une dimension physique. Le corps du poème connait lui aussi de nombreuses variations dont les mouvements les plus importants concernent essentiellement la présentation du poème et sa versification. En effet, à une exception près, le poème apparait toujours dans un premier jet haché, hachuré, haletant. Or, il faut se rappeler, pour comprendre les modifications apportées par la suite par Bauchau, de la préséance des formes sonores, vocales et rythmiques comme le précise Geneviève Henrot. À la verticalité première du poème Henry Bauchau préfère substituer la sinuosité reliante. Ce changement de forme est dicté principalement par le passage de vers courts, voire très courts au verset. En choisissant cette forme poétique, Bauchau regroupe en quelque sorte les éléments d'un corps, il remembre son poème. Or, l'apparition du verset se fait dans le mouvement paradoxal d'abandon de certains aspects narratifs présents initialement. La majorité des poèmes sauf «Les pieds sur terre» connaissent cette évolution. Ainsi, «Les mains limpides » multiplient les retours à la ligne dans sa version manuscrite, tandis que dans sa version définitive, le poète regroupe plusieurs vers pour n'en faire qu'un seul. Ainsi, nous passons de :

\author{
Je n'aurai pas vécu \\ dans la bénédiction \\ de l'outil \\ Je n'aurai vu que les reflets \\ de la lumière \\ antérieure \\ et le reflux du temps \\ de la proximité \\ Je n'ai pas obéi \\ à ma fertilité \\ Je n'ai pas consenti \\ à la sainteté \\ des matières (A394)
}

en 13 vers à : 
Je n'aurai pas vécu dans la bénédiction de l'outil.

Je n'aurai vu que les reflets de la lumière et le reflux du temps de la proximité.

Je n'ai pas consenti à ma fertilité $[\ldots]$

en seulement 3 vers. À de nombreuses reprises, la réunion de plusieurs vers en un seul s'accompagne de l'effacement de l'aspect narratif de ces vers courts. Les marqueurs de temps, les conjonctions de subordination, tous les éléments grammaticaux de liaison et d'organisation textuels sont supprimés pour leur préférer la juxtaposition. Ainsi, dans la version finale du "convive d'octobre », la subordonnée circonstancielle de temps «Lorsque j'errais, perclus par tout ce droit romain / j'avais un corps, j'étais son maître » disparait au profit d'une série de groupes compléments juxtaposés. De même, dans «Les pieds sur terre », seul poème qui connait le mouvement inverse : passage de vers longs à des vers très courts, subit malgré tout la même éviction linguistique. Les verts courts miment sur la page la posture de l'homme, «l'édifice vertical» (HD, p. 154) auquel Henry Bauchau destine le poème. Cette transformation reste donc cohérente. Toutes les subordonnées initiales de comparaison sont supprimées. De la même façon, dans «L'entreprise du sommeil», la subordonnée de temps «Quand je ne suis personne » est très vite raturée dès le premier tapuscrit (A479). L'allongement et le regroupement des vers et l'effacement des liens syntaxiques pourraient paraittre contradictoires, mais deviennent complémentaires et significatifs de la liaison dans la déliaison bauchalienne. La conscience de la diction reliante poétique offre l'ouverture déliante de la parole.

\section{Le poème-corps}

Le jeu de mots pourrait être facile aussi du côté du critique s'il déclinait à l'envi corps du poème, poème-corps et s'il n'envisageait la fabrication physique du poème comme mimétique de la corporéité dans le poème. Henry Bauchau fait de l'inconscient le siège de sa création poétique, mais son élaboration devient matérielle dans le poème. Henry Bauchau vit la création poétique corporellement, ce dont le poème se fait l'écho. 


\section{Le flux et le reflux}

Le rythme vital du poète se détecte facilement dans le poème par les mouvements de flux et de reflux qui parsèment ses textes. L'anaphore et l'hypozeuxe, deux figures de style privilégiées lors des différentes étapes de travail des manuscrits miment le retour du même, la sinuosité attendue par Bauchau. Ainsi, dans «Le convive d'octobre », les retours à la ligne et les rassemblements de plusieurs vers en un seul mettent en valeur l'anaphore en "Je suis » avec : "Je suis né, je suis relié à l'amitié de la matière / Je suis peuplé, je suis convié / Je suis le convive du corps » (HD, p. 150). L'effet mémoriel et visuel est d'autant plus efficace auprès du lecteur que l'anaphore est externe : elle existe sur les trois vers et elle est interne, puisqu'elle se prolonge à l'intérieur même des deux premiers vers. De même, dans «Les inscriptions », l'effacement de la narrativité favorise l'anaphore en "Argile » et associe la matière à la création. Les hypozeuxes des «Paumes» et de «La guêpe» avec pour le premier l'alternance anaphorique de « Va, toi » et de «tu es » et pour le second la succession des participes passés adjectifs contribuent à donner une corporéité verticale au poème.

Le changement de focus accompagne le mouvement du regard du corps vers le paysage. Les versions successives de plusieurs poèmes manifestent cette mobilité de l'attention et le déplacement du point d'intérêt. Si initialement elle se centralise sur le Je, elle se détourne très rapidement vers le corps-monde pour en souligner les accointances et les résonances avec celui-ci. À un homme statique et emprisonné succède un poète mobile et en mouvement. La marche du poème dans ses multiples versions transfigure les déplacements imaginaires du poète. Ce sont même des termes qui circulent d'un manuscrit à un autre comme « ensemencée » et « dénouée. Le premier terme apparaît initialement dans la première version des «Genêts ${ }^{7}$ (A433) et lui est préféré finalement «dénouée ». «Et dans l'oreille ensemencée » devient «Et dans l'oreille dénouée ». Or, «ensemencée » est l'adjectif qui apparait au fil des nombreuses versions de «La guêpe» dénommée initialement "L'imprononcé » (A461) avec « une terre nouvelle toujours ensemencée » (HD, p. 159). Le transfert d'un terme aussi significatif

\footnotetext{
${ }^{7}$ Le titre est alors « Minime ».
} 
entre deux poèmes montre l'évolution de l'ouverture poétique : l'oreille du poète qui ne voulait pas entendre avait besoin d'être dénouée avant d'être féconde. Les glissements de terme peuvent être internes à un poème comme dans «La goutte d'eau » pour lequel Bauchau préfère « Le plus ancien des mots / Glisse comme un regret des fêtes sous-marines » (HD, p. 159) à

Frileusement sur un fil de coton

D'un long regard glissant entre les cils de neige

Le plus ancien des mots

Surgit comme un regret des fêtes sous-marines (A454)

Le choix de faire du participe présent « glissant» le verbe principal de la phrase aux dépens de « surgit » inscrit la perpétuité du mouvement audelà de la fin du poème. Le verbe "surgir» indique un mouvement brusque, soudain et limité dans le temps, contrairement à "glisser» qui trace le sillon du poète dans la durée et sur la page. Ce mouvement s'inscrit également dans l'évolution des «Pieds sur terre » où l'accent n'est plus mis sur les pieds comme avec le manuscrit A410 et ces quelques vers :

Par la jeunesse des foules $[\ldots]$

Amis des pas $[\ldots]$

De la jeunesse des foulées

Danseurs trouvères

Jubilateurs

Des pas de la petite enfance

mais sur le mouvement des pieds avec

Enfants nouveaux ne craignez rien

de la jeunesse des foulées

Ils ont les pieds sur terre

nos pas

nos paradis

L'écholalie finale mime le travail du poète marcheur et le mouvement de la création.

Tous ces mouvements corporels sont en définitive le corollaire des flux sanguins et respiratoires du corps. Le choix poétique du verset en est un des reflets; la longue inspiration qui le guide est plus significative que les halètements des vers courts. "Les mains limpides» et «Les 
paumes » présentées de manière condensée comme c'est le cas dans l'édition de 1995 intensifient la concordance entre le mouvement du corps, son rythme et celui de la terre. Le «reflux du temps», «ma fertilité » sont ainsi accolés aux "privilèges du parleur» et des «propriétés du texte ». De la même façon, c'est dans l'origine de toute vie qu'il faut chercher la genèse du poème comme nous l'indique le premier titre de «Cellules »: «Préhistoire». L'avant poème revient à notre part infime qui contient originellement toutes nos informations génétiques et qui se différencie progressivement pour n'en exprimer que quelques-unes. Le passage de "Cellule» au singulier à la forme pluriel participe du même mouvement. La respiration du poème se voit par les indécisions du poète et les allers-retours entre deux formulations comme dans «Les mains limpides» (A398) et son hésitation entre les verbes «voir » et « connaitre » et entre le passé composé et le futur antérieur. La version finale favorisera le verbe voir au futur antérieur. Le poète y construit l'origine de sa poésie: la vue, mais des poèmes et recueils ultérieurs développeront davantage cette préséance créatrice.

\section{Le lieu et le temps}

Le poète vit corporellement la création, tout en faisant du paysage son siège. Le temps et l'espace deviennent très fréquemment le révélateur et le germe du poème qui doit se calquer sur eux. C'est ainsi que dans «L'entreprise du sommeil », c'est dans «le paysage du corps » (HD, p. 161) qu'est à lire finalement le poème ou encore que dans «L'ardoise » c'est «la maison du corps» (HD, p. 151) qu'édifie le poète avec son texte. L'alchimie élémentaire représente l'enjeu de sa création. La symbiose entre le corps du poème, du poète et celui du paysage s'élabore progressivement comme nous le rappellent "Le convive d'octobre», «Les mains limpides", "Les paumes», "Les mots perdus» ou «La signature ». Dans le premier tapuscrit du «Convive» (A375), plusieurs formules soulignent l'association désirée entre le corps et le paysage. Ce ne sont pas moins de douze vers qui insistent sur cette construction difficile avec :

\footnotetext{
J'avais un corps, j'étais son maitre

Je n'avais rien et rien ne fut réel

Sinon que je suis là, dans mon corps de pensée

Et que je suis d'abord du monde.

Je vis dans le pays du don
} 
Hormis l'erreur, rien ne me sépare de rien.

Je suis né, je suis relié

À l'amitié de la matière.

Je suis peuplé, je suis convié

Je suis le convive du corps

Il est le convive du monde.

Toute l'argile déliée

Écoute son érable rouge.

Or, dans la version finale, le poète est inclus dans le monde avec l'anaphore du verbe "être » et "d'être dans l'océan plongeur, baleine franche / d'être le sel perdu dans l'océan du corps». Ces deux vers précèdent les quelques vers cités précédemment qui deviennent euxmêmes : "Je suis né, je suis relié à l'amitié de la matière / Je suis peuplé, je suis convié / Je suis le convive du corps » (HD, p. 150). C’est ce que souligne le glissement du rapport de possession entre le pays et ses habitants dans «Les paumes » où "Ton Amérique de couleurs où l'on défriche les Indiens » (A405) devient "L'Amérique de couleurs où l'on défriche les Indiens» (A405) et finalement "C'est l'Amérique où l'on défriche tes Indiens» (HD, p. 153). La version finale des «Mots perdus » apparie les éléments au corps quand le tapuscrit (A450) original les juxtaposait. «Ange », « argile », « le livre moi-même », « neige », «verre » et «le livre du ciel » se succédaient quand dorénavant la neige, le verre et le livre du corps s'équivalent.

La dissolution cosmique du poète favorise la création et se fonde sur sa complète ouverture au monde. Elle est progressive dans «Les inscriptions » puisque si au départ le poète ne fait que "se souvenir » comme dans le manuscrit A429 avec l'anaphore «je me souviens», il devient mémoire du cosmos dans la version finale avec son enveloppement par les éléments constitutifs. Les suppressions du «Second souffle» et des «Pieds sur terre» ou des "Inscriptions» déplacent l'attention et l'importance du corps, du je vers le monde. Si « Je » et «le corps » sont répétés à l'envi dans le premier tapuscrit (A438) $\mathrm{du}$ «Second souffle » avec «corps/pesant», « corps ombilical» «corps subtil» et «peuple animal et animé du corps», toutes ces mentions disparaissent complètement dans la version publiée au profit des «poumons », du « ciel» et du « souffle ». De même, dans «Les pieds sur 
terre ", l'insistance sur le pied et tous ses composants : talon, plante... est remplacée par la communion du pied avec la terre.

Cette dissolution corporelle dans le paysage n'est effective que par une modification de l'ordre temporel. Il s'agit pour le poète nageur de remonter le cours du poème, de revenir aux origines pour dissoudre la chronologie. C'est en ce sens que nous devons interpréter l'hésitation entre passé composé et futur antérieur dans «Les mains limpides ». Le choix des temps verbaux dans la suite du poème conforte cette piste. Le poète passe du futur antérieur au passé composé, au passé simple, au présent pour finir par des phrases averbales, moment où le poète est «délié avec» dans le paradoxe de son écriture. Dans d'autres poèmes, comme «Le convive d'octobre» ou «Les jambes vives», le présent omnitemporel, symbole du suspens du temps est généralisé dans la version finale. L'homme doit faire corps avec le paysage pour devenir poète et favoriser l'alchimie élémentaire à l'origine du processus créateur. Les nombreuses hésitations bauchaliennes miment en définitive le mouvement fondateur de l'unité poétique de cette œuvre: davantage de reliance dans la déliaison. C'est ce que signifie l'utilisation fréquente du verbe « être » en copule comme dans « La guêpe » (HD, p. 159).

\section{L'absence significative}

C'est dans le retrait et dans la suppression que réside le sens du poème. Avec Bauchau, l'absence devient significative. Le travail de création du poète est de se retirer pour laisser éclore le poème, ce qui se traduit physiquement dans les manuscrits et les tapuscrits par les nombreuses suppressions qu'il y effectue. Il lui faut retirer ce qui peut faire sens immédiatement. Le retrait du poète est essentiel pour que le poème émerge. Dans «Le convive», c'est à la mort du Je que nous assistons au fil des différentes versions. Le poète s'efface et efface son corps du poème pour mieux le révéler paradoxalement dans le monde, dans la version finale. "L'arbre bleu » intitulé initialement "Un arbre » (A425) puis «Versant de l'ombre» (A426) affiche une présence paradoxale du Je. S’il est absent du premier tapuscrit - le poète nous raconte l'histoire d'un arbre et d'un «enfant d'hiver» (A425) - dans la version finale, Je apparaît effectivement, mais dans le contexte mortifère de «l'ombre », des «doigts bleuis» et du vide de l'écho de sa parole. L'effacement et la disparition du Je, mouvement majeur de la création 
bauchalienne participe à l'osmose cosmique que le poète appelle de tous ses vœux. Le retrait du poète est multifocal : il est énonciatif, poétique et parfois lyrique. L'effacement de soi permet une meilleure présence au monde poétique.

\section{Réduction des poèmes}

Cet effacement du Je va de pair dans le processus créateur avec la réduction que Bauchau fait subir à tous ses poèmes. Le geste quasi permanent dans les différents états des poèmes, au-delà des modifications ponctuelles de termes, ce sont certainement les suppressions nombreuses et importantes. Ces disparitions sont de plusieurs ordres : elles existent entre un manuscrit et un tapuscrit, de tapuscrit à tapuscrit, de tapuscrit à l'édition de 1974, de celle-ci à l'édition de 1986, et de l'édition de 1995 chez Labor à l'édition de 2009 chez Actes Sud. L'ordre des poèmes connait également un bouleversement important entre les deux premières éditions. Des poèmes entiers sont également touchés par ces suppressions puisque dans l'édition de 1974 deux poèmes ne seront jamais repris dans les versions ultérieures : «La sourde oreille» et «Le ciel prodigue $»^{8}$ et qu'entre les deux dernières éditions, "Les genêts », "L'enfant de sel » et «Cellules » ne sont pas conservés non plus. Les poèmes les plus emblématiques de ce mouvement sont certainement «Les pieds sur terre» et «Le second souffle ». Le premier passe d'une centaine de vers manuscrits à 35 vers dans la version finale, de même pour le second texte, des 52 vers originels il ne reste que les 14 derniers dans la version finale. Les modifications de termes ou de syntaxe que nous avons observées précédemment ont la même vocation à signifier dans l'absence. «Le convive », «Les mains limpides », «Les inscriptions », «La goutte d'eau » ou encore «Les cellules » en sont les parfaits exemples, tous ces poèmes connaissent des suppressions importantes qui rendent la portée du poème beaucoup plus implicite. Dire sans dire serait la gageure poétique de Bauchau, signifier par le silence, le blanc ou la syllepse devient la caractéristique stylistique de son œuvre. Le travail sur les états des manuscrits ou des tapuscrits montre un auteur qui enlève consciemment les outils de la signification. La formulation ramassée du vers 14 des «Paumes» avec «le monde est un désir et le terrible est sa lumière »

\footnotetext{
${ }^{8}$ Certains éléments du manuscrit A430 pourraient être lus comme les prémices de ce poème.
} 
(HD, p. 153) est éclairée dans les tapuscrits (A405 sqq) par un vers qui le précède : «Le monde n'entre pas dans cet horizon d'arbres ». Or, ce vers effacé apporte des clés pour comprendre le vers suivant. De même, dans l'édition de 2009, le poète supprime les quatre derniers vers de la première strophe :

Plus loin fut le rêve des pauvres

Lorsque la terre est rouge et le cœur blanc

C'est l'apocalypse du Noir

C'est l'Amérique où l'on défriche tes Indiens (HD, p. 158.)

Ces quatre vers identifiaient le référent, certes imaginaire à entendre dans les termes génériques, nombreux dans ce poème. La disparition des quatre premiers vers initiaux de «L'attentive» (tapuscrit A450) devenue «Les mots perdus » remplace la structure en hypozeuxe du poème par une construction en chiasme dont le centre de symétrie est le vers définitoire: "tout est écrit dans le livre du corps» (HD, p. 158). L'effacement des deux premiers vers: «Frileusement sur un fil de flocons / D'un long regard glissant entre les cils de neige » qui différaient le véritable enjeu du texte, accentue la portée herméneutique du poème. Le procédé est le même dans "Cellules", les quatorze premiers vers se condensent en deux vers dans la version finale qui ne conserve que le lien entre l'écoute intérieure et la construction du poème.

\section{Le rôle du lecteur}

Mais si le poète favorise la dimension exégétique de ses poèmes, c'est peut-être pour laisser une place plus importante à son lecteur, pour signifier à ce dernier l'importance d'une construction du sens à plusieurs mains, à plusieurs voix et surtout à plusieurs consciences. Ce dernier, comme le poète au moment de la création doit être capable de recevoir le poème quand le poète, lui, le subit. L'effet de concentration du sens laisse plus d'espace d'interprétation au lecteur tout en ôtant au poème son caractère référentiel. Sans l'interpeller directement dans le poème ou le faire apparaitre concrètement, Bauchau laisse un espace dans lequel il se glisse facilement. Les clés de la construction poétique données dans «Les pieds sur terre » et « La guêpe » apportent quelques éléments d'aide au lecteur, mais c'est aussi la modification apportée aux derniers vers des «Genêts" (A433) qui donne la disposition dans laquelle le lecteur doit, lui aussi, se trouver. Le remplacement de « et dans l'oreille ensemencée / 
Il n'y a que leur voix minime / et leur son savoir de graine» par «Et dans l'oreille dénouée / Le son de leur savoir de graine » déplace l'origine de la parole et le rapport au monde. Le poète interprète le monde et lit son corps comme le lecteur de Bauchau est amené à devenir l'exégète du poème. L'obscurité apportée par les suppressions laisse finalement plus de place et plus de liberté au lecteur.

\section{Le passeur architecte}

La figure du poète apparait alors en passeur qui transmet la parole du monde. Il fait transiter les bruissements du corps et du monde pour faire émerger le corps du poème. Être passeur ne l'empêche pas d'être également un architecte pour qui la construction du poème fait sens. En témoignent les nombreux déplacements grammaticaux et la préférence accordée à la syllepse grammaticale comme dans "L'arbre bleu » où l'apparition du groupe nominal «de cet arbre sauvage» (HD, p. 156) est retardée pour brouiller son rapport probable de dépendance avec «la voix ».

Le remembrement du corps se fait paradoxalement dans l'effacement de sa personne, ce qui apporte du liant dans la déliaison du poème. Le corps en blason de Bauchau n'invalide pas le rôle d'architecte qu'il tente de tenir dans sa poésie. Le poème doit être construit malgré son origine obscure. C'est exactement ce qu'il met en œuvre dans le parcours italien de «La Dogana » avec «L'architecture ». Il s'agit certes de célébrer la beauté artistique de Venise, mais c'est aussi une invitation à y lire la propre fabrique de son poème. Si nous suivons les recettes qu'il y donne : il faudrait être en éveil pour accueillir la femme et faire surgir des profondeurs «l'architecture / qui mène par les fondements» ( $H D$, p. 201). Quelques vers épars non publiés en l'état soulignent également cette tentation poétique :

Du sang du corps entier

D'une langue d'amour

Une parole primevère

Une méditation de l'air

Une écriture de vertige

Une parole de prodige

D'un vocable vertical

L'architecture de la joie (A430) 
Écrire, c'est construire une tour verbale, c'est l'élaborer et en concevoir les plans. Les mouvements de clôture et d'ouverture que subissent les poèmes dans leurs différentes versions signalent cette volonté de construire un édifice verbal. Ainsi, la suppression des vers médians dans «Le convive d'octobre » renforce la collusion entre les deux premiers vers et la fin du dernier vers où l'érable rouge est la réunion de l'archange rouge, de l'érable et du mélèze du début. C'est le tout qui fait sens dans la dispersion des éléments. Il en est de même dans «Les paumes» selon un mouvement inverse puisque la suppression du troisième vers «Et le cœur est veillé dans ce jardin d'ardoise » (A404) qui entrait en résonance avec le dernier «De ce pays d'ardoise où le cœur est gardé » efface la clôture bancale du poème au profit d'une autre forme de clôture avec la triade corps-esprit au début du poème et cœur en sa toute fin. C'est également ainsi qu'il faudrait analyser la préférence de la juxtaposition à l'articulation des phrases verbales dans «La guêpe ». La venue de la parole est un flux qui commence sans notre accord et qu'il faut savoir relier à l'ensemble pour en comprendre le sens. La disparition de la matière propice à la parole poétique, l'argile dans l'édition de 2009 participe de ce même mouvement : il met en acte ce qu'il veut signifier. Il ne s'agit plus de fixer son attention sur ce qui permet le poème, mais sur le résultat de cette ouverture : l'édifice vertical et verbal. L'unité poétique de l'œuvre de Bauchau se fonde sur la reliance dans la déliaison.

Centraliser notre attention sur le corps du poète dans les manuscrits dans un recueil particulier a permis d'affiner l'élaboration corporelle que nous avions subodorée dans l'ensemble de son œuvre poétique antérieurement. La marche du poète donne le mouvement de va-et-vient du poème dans ses différentes publications. La condensation poétique permet l'élaboration architecturale du poème dans les blasons du corps.

Marianne FroYe Université de Cergy-Pontoise 